\title{
Zero energy mode for an electron in graphene in a perpendicular magnetic field with constant asymptotics
}

\author{
Juan Sebastián Ardenghi ${ }^{\dagger \ddagger}$, Alfredo Juan ${ }^{\dagger \ddagger}$, Valeria Orazi ${ }^{\dagger \ddagger}$, Lucas Sourrouille ${ }^{\dagger \ddagger a}$, \\ †Departamento de Física, Universidad Nacional del Sur, Av. Alem 1253, \\ B8000CPB, Bahía Blanca, Argentina \\ ${ }^{\ddagger}$ Instituto de Física del Sur (IFISUR, UNS-CONICET), Av. Alem 1253, \\ B8000CPB, Bahía Blanca, Argentina \\ ${ }^{a}$ sourrou@df.uba.ar
}

February 1, 2019

\begin{abstract}
We study the influence of a perpendicular magnetic field with the asymptotics $B(r \rightarrow \infty)=B_{0}$ in a electrons in graphene. It is shown that the zero-energy solutions can exist only for one pseudospin direction, depending on the sign of the magnetic field in the infinite boundary. This, shows that zero-energy level is robust with respect to possible inhomogeneities of the magnetic field. In addition, we show that the number of the states with zero energy for one pseudospin projection is infinity. These results should be useful in the study of ripples which cause a scattering of Dirac particles in slowly decreasing magnetic fields where the asymptotic states is easy to define.
\end{abstract}

\section{Introduction}

The energy spectrum of fermions coupled to gauge fields presents an important property, the existence of zero-energy modes equally shared by electrons and holes. This is a consequence of one of the most important theorems of modern mathematics, the Atiyah-Singer index theorem [1, 2]. This theorem has important applications in quantum field and superstring theories [3, 4]. It, also, has important consequences on graphene [5, 6] where the anomalous (half-integer) quantum Hall effect in single-layer graphene is a consequence of the Atiyah-Singer theorem. In addition, a similar statement has been proven for the anomalous quantum Hall effect in graphene bilayer [7, 8]. The existence of the zero-energy states for fermions in inhomogeneous magnetic fields in two dimensions was demonstrated explicitly by Aharonov and Casher [9]. They, was 
able to show that the ground state is exactly calculable and possesses a degeneracy related to the total magnetic flux. Specifically, they suppose the case of an infinite sample with the magnetic flux $\Phi$ localized in a restricted region and showed that zeroenergy solutions can exist only for one spin direction, depending on the sign of the total flux. Also, they showed that the number of the states with zero energy for one spin projection is equal to $N$, where $N$ is the integer part of $\frac{\Phi}{2 \pi}$.

In this note we generalize the idea of Aharonov and Casher and study electrons in graphene under the influence of perpendicular magnetic field with the asymptotics $B(r \rightarrow \infty)=B_{0}$, where $B_{0}$ is a real constant. Particularly, we show that the zeroenergy solutions can exist only for one pseudospin direction, that in graphene implies that the wave function of electrons are not vanishing only for one sublattice basis and in turn this depends on the sign of the magnetic field in the boundary. In addition, we show that the number of the states with zero energy for one pseudospin projection is infinity. These results are importnat in nanophysics, because it is expected that graphene will serve a base for new optoelectronic devices ([5], [10]). In particular, in order to control the motion of electrons, the use of non-homogeneous magnetic fields has been study, through the deposit of nanostructured ferromagnets ([11, [12], [13]). In recent works ([14], [15], [16], [17] and [18]), the magnetic properties of graphene under constant perpendicular magnetic field taking into account the Zeeman effect and Rashba spin-orbit coupling has been studied, showing that zero energy states alter drastically the magnetic oscillations and the quantum Hall effect. In order to extend to the variable magnetic fields, we consider a constant asymptotics for the magnetic field that model a magnetic antidot, where $B$ is zero in a circular region and nonzero outside this region which can be achieved by having a magnetic vortex piercing the graphene layer [13. This work will be organized as follow: In section II, the simplified model for graphene in a constant asymptotic non-vanishing magnetic field is introduced. In section III, the solutions ar computed and discussed- The principal findings of this paper are highlighted in the conclusion.

\section{The model}

Let us start by considering electrons in graphene in the long wavelength approximation, where the $(2+1)$-dimensional Dirac-Weyl model whose Hamiltonian is described by

$$
H=\left(\sigma_{x} p_{x}+\sigma_{y} p_{y}\right)
$$

Here, the $\sigma_{i}(i=x, y)$ are $2 \times 2$ Pauli matrices acting in the sublattice basis, i.e.

$$
\sigma_{x}=\left(\begin{array}{cc}
0 & 1 \\
1 & 0
\end{array}\right), \quad \sigma_{y}=\left(\begin{array}{cc}
0 & -i \\
i & 0
\end{array}\right)
$$

$p_{i}=-i \partial_{i}$ is the two-dimensional momentum operator and for simplicity we have considered that the Fermi velocity $v_{F}=1$. The massless Dirac-Weyl equation in $(2+1)$ dimensions is

$$
\sigma^{i} p_{i} \Psi(x, y, t)=i \partial_{t} \Psi(x, y, t)
$$


Here, $\Psi(x, y, t)$ is the two-component spinor

$$
\Psi=\left(\psi_{A}, \psi_{B}\right)^{T}
$$

here $\psi_{a}$ and $\psi_{b}$ represent the envelope functions associated with the probability amplitudes in each sublattice basis. Since, we are interested in stationary states, it is natural to propose a solution of the form

$$
\Phi(x, y, t)=e^{-i E t} \Psi(x, y)
$$

then, the time-independent Dirac-Weyl equation is

$$
\sigma^{i} p_{i} \Psi(x, y)=E \Psi(x, y)
$$

In the presences of a perpendicular magnetic field to the $(x, y)$-plane, we replace the momentum operator $p_{i}$ by the covariant derivative, defined as $D_{i}=-i \partial_{i}+A_{i}(i=x, y)$, where $A_{i}$ are components of the vector potential, such that

$$
B(x, y)=\partial_{x} A_{y}-\partial_{y} A_{x}
$$

where $B(x, y)$ is the transversal magnetic field. Thus, the equation (4) becomes,

$$
\sigma^{i} D_{i} \Psi(x, y)=E \Psi(x, y)
$$

We can develop this equation to get

$$
\left(\begin{array}{cc}
0 & D_{1}-i D_{2} \\
D_{1}+i D_{2} & 0
\end{array}\right)\left(\begin{array}{l}
\psi_{A} \\
\psi_{B}
\end{array}\right)=E\left(\begin{array}{l}
\psi_{A} \\
\psi_{B}
\end{array}\right)
$$

where $\psi_{A}$ and $\psi_{B}$ are the sublattice components of the spinor $\Psi$ (i.e. $\Psi=\left(\psi_{a}, \psi_{b}\right)^{T}$ ). From this equation we can write the two coupled equations for the components $\psi_{A}$ and $\psi_{B}$

$$
\begin{aligned}
& {\left[D_{1}-i D_{2}\right] \psi_{B}=E \psi_{A}} \\
& {\left[D_{1}+i D_{2}\right] \psi_{A}=E \psi_{B}}
\end{aligned}
$$

Here, we are interested in the zero energy modes. This solution may be constructed explicitly following, the work done by Aharonov and Casher [9]. Thus, we can introduce a scalar potential $\lambda(x, y)$ such that,

$$
A_{x}=-\partial_{y} \lambda, \quad A_{y}=\partial_{x} \lambda
$$

and due to the equation (5),

$$
B=\partial_{x}^{2} \lambda+\partial_{y}^{2} \lambda
$$

Then, the equations (8) and (9), for the energy zero case, can be written in the form

$$
\begin{aligned}
& {\left[\left[-i \partial_{x}-\partial_{y}\right]+\left(-\partial_{y} \lambda-i \partial_{x} \lambda\right)\right] \psi_{B}=0} \\
& {\left[\left[-i \partial_{x}+\partial_{y}\right]+\left(-\partial_{y} \lambda+i \partial_{x} \lambda\right)\right] \psi_{A}=0}
\end{aligned}
$$


The potential $\lambda(x, y)$ can be excluded by the substitution

$$
\psi_{A, B}=f_{A, B} e^{\gamma \lambda}
$$

where $\gamma=1$ and -1 for $\psi_{A}$ and $\psi_{B}$ respectively. Then the equations (11) and (12) transforms into the equation

$$
\left[-i \partial_{x}+\gamma \partial_{y}\right] f_{A, B}=0
$$

Thus, $f_{A}$ and $f_{B}$ are analytic and complex conjugated analytic entire functions of $z=i x+y$, respectively.

For a transversal magnetic field $B(x, y)$ with the asymptotics $B(r \rightarrow \infty)=0$ the problem was addressed by Aharonov and Casher in Ref.[9], and leads to zero-energy solutions exist only for one spin direction, depending on the sign of the total magnetic flux. Also they proved that the number of the states with zero energy for one ospin projection is equal to $N$, where $N$ is the the closest integer to the total flux in units of the flux quantum.

In this letter we are interested in magnetic fields with the asymptotics $B(r \rightarrow \infty)=B_{0}$, where $B_{0}$ is a constant real number different from zero. Hence, as $r \rightarrow \infty$ the equation (5) becomes,

$$
B_{0}=\partial_{x} A_{y}-\partial_{y} A_{x}
$$

which can be solved by

$$
A_{x}=-\frac{B_{0}}{2} y, \quad A_{y}=\frac{B_{0}}{2} x
$$

which coincides with the symmetric gauge. To proceed, we can obtain an expression for $\lambda(x, y)$ by using (10) and (16),

$$
\lambda(r \rightarrow \infty)=\frac{B_{0}}{4}\left(x^{2}+y^{2}\right)=\frac{B_{0}}{4} r^{2}
$$

Then, the solution of Eq. (13) at large distances has the asymptotics, with

$$
\psi_{A, B}=f_{A, B} e^{\gamma \frac{B_{0}}{4} r^{2}}
$$

Since the entire function $f_{A, B}(z)$ cannot go to zero in all directions at infinity, $\psi_{i}$ can be normalizable only assuming that $\gamma \frac{B_{0}}{4}<0$; that is, zero-energy solutions can exist only for one spin direction, depending on the sign of the magnetic field in the boundary. Here, it is important to remark that our result remains robust with respect to possible inhomogeneities of the magnetic field in a finite region of the plane. In other words that means that the spin polarization, depends only on asymptotic behavior of the magnetic field. This statement is important for real graphene since the effective magnetic field there should be inhomogeneous due to the effect of so-called ripples [5], [6].

To conclude this section we can count how many independent solutions of Eq. (14) we have. As a basis, we can choose just polynoms searching the solutions of the form

$$
\psi_{b}=z^{j} e^{-\frac{B_{0}}{4} r^{2}}
$$


(to be specific, we consider the case $B_{0}>0$ ), where $\mathrm{j}=0,1,2$, . . Since $e^{-\frac{B_{0}}{4} r^{2}}$ decays faster than any power of $z$. One can easily see from Eq. (17) that the solution is integrable with the square for any value of $j$. Therefore, the number of the states with zero energy for one spin projection is infinity, and there are no such solutions for another spin projection.

\section{Results and discussion}

Let us, now, discuss the applications of our result to graphene systems. In graphene, the spin polarization is a pseudospin polarization, that is reflected in a non-vanishing envelope function only for the $A$ or $B$ sublattice of the Bravais lattice of graphene depending on the sign of $B_{0}$. Indeed, graphene has a degeneracy related to the Dirac points in the reciprocal lattice that introduces the valley index $\tau= \pm 1$, where +1 is for the $K$ valley and -1 for the $K^{\prime}$ valley, where $K$ and $K^{\prime}$ are two inequivalent Dirac points in $k$-space ([19], [20]). The Hamiltonian can be written as $H=\tau_{z} \otimes$ $\left(\sigma_{x} p_{x}+\tau \sigma_{y} p_{y}\right)$ where $\tau_{z}$ is the $\sigma_{z}$ operator in the valley subspace. Following this, the wavefunction can be written as $\psi_{A, B}=f_{A, B} e^{\tau \gamma \lambda}$. Then, for $B_{0}>0$ in the $K$ valley only the $B$ sublattice component is non-vanishing and in the $K^{\prime}$ valley only the $A$ sublattice is non-vanishing, that is, the case of zero energy is special since its amplitude is nonzero only in one of the sublattices, namely, at $B$ sites for $K$ and $A$ sites for $K^{\prime}$. Due to the degeneracy of the Hamiltonian and when no other external mechanism is consider to break the valley symmetry of the Hamiltonian, the zero mode solution consists in a superposition in the pseudospin space

$$
\begin{aligned}
\left|\Psi_{+}\right\rangle & =\frac{1}{\sqrt{2}} e^{-\frac{B_{0}}{4} r^{2}}\left(f_{B K}|B\rangle|K\rangle+f_{A K^{\prime}}|A\rangle\left|K^{\prime}\right\rangle\right) \\
\left|\Psi_{-}\right\rangle & =\frac{1}{\sqrt{2}} e^{\frac{B_{0}}{4} r^{2}}\left(f_{A K}|A\rangle|K\rangle+f_{B K^{\prime}}|B\rangle\left|K^{\prime}\right\rangle\right)
\end{aligned}
$$

where $\left|\Psi_{+}\right\rangle$is for $B_{0}>0$ and $\left|\Psi_{-}\right\rangle$is for $B_{0}<0$. The entanglement between the valley and pseudospin is manifest and can be tuned with the magnetic antidot by alternating the asymptotic behavior of the magnetic field. In turn, $\left\langle\Psi_{+} \mid \Psi_{-}\right\rangle=0$ and no transition between zero mode solutions is possible. In order to obtain the number of independent solutions, the differential equation for $f$ is in eq. (14) only for the $K$ valley, although by symmetry, $f_{A K^{\prime}}=f_{B K}=\bar{z}^{n}, f_{B K^{\prime}}=f_{A K}=z^{n}$ can be obtained. For single valuedness, the function $f$ has to be a polynomial, which implies that $f$ must be of degree $n$, where $n$ is determined by the total flux $\Phi=\int B d A=(n+\epsilon) \phi_{0}$ where $\phi_{0}=\hbar / e$ is the quantum of magnetic flux and $0<\epsilon<1$. Then the Hamiltonian for each valley has therefore $n$ zero-modes independently of the shape of the magnetic field in the interior.

In this sense, graphene with constant asymptotics magnetic field has a chiral symmetry in the massless limit of the Dirac equation, except for the zero modes. Should be stressed that the symmetry in the Hamiltonian in graphene is a manifestation of supersymmetry [21] in quantum mechanics, which appears as a quantum Hall spectrum 
in multilayer graphene [22] and is robust for arbitrary magnetic fields. In particular, for random magnetic fields, as it can occur with ripples [23], Dirac particles scatters slowly decreasing magnetic fields whose asymptotics can be positive or negative randomly and the scattering states can be represented by eq.(18) with arbitrary spacedependent $f$ coefficients. Consequently, while a random on-site disorder potential gives only intravalley mixing within either the $K$ and $K^{\prime}$ valleys, random hopping can cause intervalley mixing. Nevertheless, for general hopping disorder potential $U$ (see eq.(7) of [24] $),\left\langle\Psi_{+}|U| \Psi_{-}\right\rangle=0$, no transition between asymptotic zero modes is allowed to first order in the random potential and intervalley mixing is expected for $n>0$ modes or for $n=0$ at second order in the random potential.

\section{Conclusions}

In summary, we have generalized the result found in Ref. [9] for the cases in which the magnetic field is different from zero as $r \rightarrow \infty$. In particular, we showed that the zeroenergy solutions can exist only for one pseudospin direction in graphene, depending on the sign of the magnetic field on the infinite edge. Thus, the zero-energy level is protected by the value of the magnetic field in boundary, which show that it is robust with respect to possible inhomogeneities of the magnetic field. Finally, we have considered the valley degrees of freedom, we habe found that zero energy mode has two orthogonal entangled solution in the valley and pseudospin states and that can be tuned with the magnetic field asymptotics.

\section{Acknowledgements}

This paper was partially supported by grants of CONICET (Argentina National Research Council) and Universidad Nacional del Sur (UNS)and by ANPCyT through PICT 1770, and PIP-CONICET Nos. 114-200901-00272 and 114-200901-00068 research grants, as well as by SGCyT-UNS., J. S. A. and L. S. are members of CONICET

\section{References}

[1] M.F. Atiyah, I.M. Singer, Ann. of Math., 87, 484 (1968) .

[2] M.F. Atiyah, I.M. Singer, Proc. Natl. Acad, Sci. USA, 81, 2597 (1984).

[3] M. Kaku, Introduction to Superstrings, Springer, New York, (1988).

[4] M. Nakahara, Geometry, Topology and Physics, IOP, Bristol, (1990).

[5] K.S. Novoselov, A.K. Geim, S.V. Morozov, D. Jiang, M.I. Katsnelson, I.V. Grigorieva, S.V. Dubonos, A.A. Firsov, Nature, 438, 197 (2005). 
[6] M.I. Katsnelson, Mater. Today, 10, 20 (2007).

[7] M.I. Katsnelson, M.F. Prokhorova, Phys. Rev. B, 77, 205424 (2008).

[8] K.S. Novoselov, E. McCann, S.V. Morozov, V.I. Falko, M.I. Katsnelson, U. Zeitler, D. Jiang, F. Schedin, A.K. Geim, Nature Phys. 2, 177 (2006).

[9] Y. Aharonov, A. Casher, Phys. Rev. A 19, 2461 (1979).

[10] Y. Zhang, Y. W. Tan, H. L. Stormer, and P. Kim, Nature London, 438, 201 (2005).

[11] F. M. Peeters and A. Matulis, Phys. Rev. B 48, 15166 (1993).

[12] J. Reijniers, F. M. Peeters, and A. Matulis, Phys. Rev. B 64, 245314 (2001).

[13] J. Reijniers, F. M. Peeters, and A. Matulis, Phys. Rev. B 59, 2817 (1999).

[14] F. Escudero, J. S. Ardenghi, P. Jasen and A. Juan, Physica B, 101, 518 (2017).

[15] J. S. Ardenghi, A. Juan, F. Escudero and L. Sourrouille, Europhysics Letters, 118, 21001 (2017).

[16] F. Escudero, L. Sourrouille, J. S. Ardenghi and P. Jasen, Superlattices Microstructures, 101, 537-546 (2016).

[17] F. Escudero, J. S. Ardenghi, L. Sourrouille and P. Jasen, J. Magn. Magn. Mater., 429, 204-298 (2016).

[18] J. S. Ardenghi, P. Bechthold, P. Jasen, E. Gonzalez and O. Nagel, Physica B, 427, 97-105 (2013).

[19] J. S. Ardenghi, P. Bechthold, E. A. Gonzalez, P. Jasen and A. Juan, Eur. Phys. J. $B, \mathbf{8 8 : 4 7}(2015)$.

[20] L.A. Wu and M. Guidry, Scientific Reports, 6, 22423 (2016).

[21] Witten E., Nucl. Phys. B, 188, 513 (1981).

[22] Ezawa M., Physica E, 40, 269-272 (2007). 
[23] M. A. H. Vozmediano, M. I. Katsnelson, F. Guinea, Phys. Reports, 496, 4-5, 109-148 (2010).

[24] A. L. C. Pereira, C. H. Lewenkopf, and E. R. Mucciolo, Phys. Rev. B, 84, 165406 (2011). 